\title{
SUMMABILITY OF FOURIER SERIES ON THE QUATERNIONS OF NORM ONE
}

\author{
BY
}

\section{GUSTAVE RABSON}

1. Introduction. The Peter-Weyl theorem asserts that if $G$ is a compact group, then the matrix elements of a complete system of inequivalent irreducible unitary representations form a complete orthogonal system of functions in the Hilbert space of complex-valued square-integrable functions on $G$. When $G$ is the circle (the real numbers modulo $2 \pi$ ), then the irreducible representations are all one-dimensional, and are, in fact, the functions $\exp (\operatorname{in} x)$. The Peter-Weyl theorem, then, makes it possible to generalize the concept of Fourier series to arbitrary compact groups and it asserts the convergence in the mean of the Fourier series of a function in $L^{2}$. Of course a sequence of functions may converge in the mean without converging at any point. For classical Fourier series there are several theorems concerning the pointwise convergence or summability of Fourier series. Two of the principal results of this type are Fejer's theorem which asserts the $(C, 1)$ convergence of the Fourier series at a point of continuity and the Fejer-Lebesgue theorem which asserts the $(C, 1)$ convergence, almost everywhere, of the Fourier series of a function in $L^{1}$. The direct analogue of Fejer's theorem has been proved when $G$ is a countable product of groups of order two by J. L. Walsh and N. J. Fine. Walsh proved the theorem in a different setting [13, Theorem VII], and the group theoretic character was pointed out by Fine $[4$, Theorem XVII].

It is the major objective of this paper to prove analogues of Fejer's theorem and the Fejer-Lebesgue theorem when $G$ is the group of quaternions of norm one. The direct analogues are no longer true but if we use the concept of the character series (see Definition 2.4) instead of the Fourier series and use $(C, 2)$ summation instead of $(C, 1)$ summation, then we obtain Theorems 9.6 and 10.1. In connection with Theorem 9.6 it is interesting to note the comment by Chandrasekharan and Minakshisundaram [2, p. 752] to the effect that for a torus of dimension $n$, at a point of continuity of $f$, the multiple Fourier series is uniformly summable $(\nu, k)$ for $k>(n-1) / 2$. It should also be pointed out that Theorem 10.1 is actually a special case of a general theorem on Jacobi polynomials [11, Theorem 9.1.4] since except for a normalizing factor the functions $(\sin n x) /(\sin x)$ (essentially the characters of the group of quaternions of norm one) are $P_{n}^{(1 / 2,1 / 2)}(y)$ where $y=\cos x$. Nevertheless it was thought that the approach followed here, being more in the spirit of classical Fourier series, would be of interest.

Presented to the Society, September 4, 1951 under the title Fejer's theorem on SU(2); received by the editors August 11, 1952. 
The author wishes to express his gratitude to Professor Hans Samelson of the University of Michigan for many helpful suggestions and for his stimulating guidance. $\mathrm{He}$ also profited from conversations with many people. In particular he is indebted to M. Jerison, J. Korevaar, and I. E. Segal. He received some financial aid from the Office of Naval Research under contract N8onr71400 during part of the time that he was working on this paper. The material in this paper has been presented to the University of Michigan in partial fulfillment of the requirements for the degree of Doctor of Philosophy.

2. Some general definitions. We shall assume that $G$ is compact and satisfies the second axiom of countability in which case it follows from the Peter-Weyl theorem that the set of irreducible representations, and therefore the set of matrix elements $\left\{m_{i\}}^{\alpha}\right\}$ (the function in the $i$ th row and $j$ th column of the $\alpha$ th representation) is countable (see [9, Theorem 22]). We shall also assume that the Haar measure has been normalized so that the measure of $G$ is one. Let us well order the set of functions $\left\{m_{v}^{\alpha}\right\}$ in any fashion, except that we must keep all the functions from a given representation together.

(2.1) Definition. By the Fourier series of a function $f$ in $L^{1}$ we mean the series

$$
\sum r_{\alpha}\left(\int_{G} f(x)\left[m_{i j}^{\alpha}(x)\right]^{c} d x\right) m_{i j}^{\alpha}(1)
$$

where $r_{\alpha}$ is the degree of the $\alpha$ th representation (which must be introduced to normalize the functions $m_{i j}^{\alpha}$ ).

It is easy to show (see [14, pp. 76-77]). that

$$
\sum_{i=1, j=1}^{r_{\alpha}} r_{\alpha}\left(\int_{G} f(x)\left[m_{i j}^{\alpha}(x)\right]^{c} d x\right) m_{i j}^{\alpha}=r_{\alpha}\left(f * \chi^{\alpha}\right)
$$

where $\chi^{\alpha}$ is the trace of the $\alpha$ th representation. We now see that the sequence of partial sums of the series $\sum_{\alpha=1}^{\infty} r_{\alpha}\left(f * \chi^{\alpha}\right)$ is a subsequence of the sequence of partial sums of the Fourier series.

(2.4) Definition. We call the series

$$
\sum_{\alpha=1}^{\infty} r_{\alpha} f * \chi^{\alpha}
$$

the character series of the function $f$.

It is clear that the convergence of the Fourier series implies the convergence of the character series although the converse is not true (as may be shown by an example).

The partial sums $S_{n}$ of the character series may, of course, be written in the form $f * \sum_{\alpha=1}^{n} r_{\alpha} \chi^{\alpha}$. In this form they resemble Dirichlet's form for the partial sums of the classical Fourier series and will, indeed, reduce to this

(1) [ ]c denotes complex conjugate. 
when $G$ is the circle and the representations are ordered in the customary way. It seems appropriate therefore to make the following definition.

(2.6) Definition. The sequence of functions $\sum_{\alpha=1}^{n} r_{\alpha} \chi^{\alpha}$ will be called the Dirichlet kernels. We shall denote them by the symbols $D_{n}$. It is easy to see that $D_{n}$ is continuous and that $\int_{G} D_{n}(x) d x=1$.

If we consider an arbitrary sequence of kernel functions, $K_{n}$, we may consider the sequence $f * K_{n}$. The following theorem is classical. We assume $K_{n}$ is in $L^{1}$ and that $\int_{G} K_{n}(x) d x=1$.

(2.7) Theorem. If $\lim \sup \int_{G}\left|K_{n}(x)\right| d x=\infty$, then for any $x$ there is a continuous function whose character series diverges for that $x$.

The classical proof given in [6, Theorem 66] applies without change.

(2.8) Definition. We call the sequence of constants $\int_{G}\left|K_{n}(x)\right| d x$ the Lebesgue constants with respect to the kernels $K_{n}$.

(2.9) Theorem. If the Lebesgue constants with respect to the kernels $K_{n}$ are uniformly bounded by the constant $C$ and if each of the functions $K_{n}$ converges to zero uniformly outside of every neighborhood of the identity, then $f * K_{n}(x)$ converges to $f(x)$ at each point of continuity if $f$ is in $L^{1}$. If $f$ is continuous, then convergence is uniform.

The following proof of Theorem 2.9 is classical. For $\epsilon>0$ we choose a neighborhood $U$ of the identity, $e$, so that

$$
\left|f\left(x y^{-1}\right)-f(x)\right|<\epsilon / C
$$

for $y$ in $U$. We have

$$
\begin{aligned}
\left|f * K_{n}(x)-f(x)\right|= & \left|\int_{G} f\left(x y^{-1}\right) K_{n}(y) d y-\int_{G} f(x) K_{n}(y) d y\right| \\
\leqq & \int_{U}\left|f\left(x y^{-1}\right)-f(x)\right|\left|K_{n}(y)\right| d y \\
& +\int_{C(U)}\left|f\left(x y^{-1}\right)-f(x)\right|\left|K_{n}(y)\right| d y \\
< & +\int_{C(U)}\left|f\left(x y^{-1}\right)-f(x)\right|\left|K_{n}(y)\right| d y .
\end{aligned}
$$

The last integral can be made arbitrarily small by choosing $n$ sufficiently large since $K_{n}$ approaches zero uniformly in $C(U)$. If $f$ is continuous, it is uniformly continuous from which it follows that convergence of the $f * K_{n}$ is uniform.

3. Generalization of theorems about central functions. The central functions are those functions of $L^{1}$ with the property that $\phi(x)=\phi\left(y^{-1} x y\right)$ for all 
$x, y$ in $G$. They can also be defined as those functions of the $L^{1}$ group algebra, $A$, of $G$ which commute with all the functions of $A$, or again as that subspace of $A$ spanned by the characters. The construction of Theorem 32 is similar to a construction used in $[14, \S 24]$.

On a compact Lie group the central functions are determined by their values on a maximal abelian subgroup. Since the maximal abelian subgroup is a torus it is often easier to work with central functions and then to generalize by means of Theorem 3.2 (see [7, Hilfsatz 3]).

(3.1) Definition. For $f$ in $L^{1}$ and $s$ in $G$ we denote by $C_{8}(f, x)$, or $C_{8}(x)$ when $f$ is clearly understood, the function $\int_{G} f\left(s t x t^{-1}\right) d t$.

(3.2) TheOREM. For almost all $s, C_{s}$ is a central function and the convergence, to $c$, of the character series of $f$ at the point $s$ is equivalent to the convergence of the character series of $C_{s}$ to $c$ at the point $e$.

Proof. Consider first the function $f^{\prime}$ on $G \times G$ defined by $f^{\prime}(t, x)=f\left(s t x t^{-1}\right)$. Since the map sending $(t, x)$ into stxt $t^{-1}$ is continuous it follows that $f^{\prime}$ is measurable. In fact $f^{\prime}$ is integrable since

$$
\begin{aligned}
\int_{G} f(x) d x & =\int_{G} \int_{G} f(x) d t d x=\int_{G} \int_{G} f(s x) d t d x=\int_{G} \int_{G} f\left(s t x t^{-1}\right) d t d x \\
& =\int_{G \times G} f^{\prime}(t, x) d(t, x) .
\end{aligned}
$$

Consequently, by Fubini's theorem the function $C_{8}(x)$ exists and is in $L^{1}$ for almost all $s$. We see also that $C_{s}(e)=\int_{G} f(s) d t=f(s)$. To complete the proof of the theorem we need only consider the fact that

$$
\begin{aligned}
\left|c-S_{n}(f, s)\right| & =\left|c-\int_{G} f(s x) D_{n}\left(x^{-1}\right) d x\right| \\
& =\left|c-\int_{G} \int_{G} f\left(s t x t^{-1}\right) D_{n}\left(x^{-1}\right) d x d t\right| \\
& =\left|c-\int_{G} C_{s}(x)\left[D_{n}(x)\right] c d x\right|=\left|c-C_{s} * D_{n}(e)\right| .
\end{aligned}
$$

4. The group of quaternions of norm one. Let us denote the group of quaternions of norm one by $Q$. It is well known that $Q$ is isomorphic to the group of $2 \times 2$ unitary matrices of determinant one and is also the twosheeted simply connected covering group of the group of proper rotations of three space. Topologically speaking $Q$ is the three sphere in four space. The characters of the irreducible representations are well known (see $[10$, p. 186] for example).

(4.1) ThEOREM. For each positive integer $n$ there is one irreducible unitary 
representation of degree $n$. The character of the representation of degree $n$ may be written in the form

$$
\sin n \phi(x) / \sin \phi(x)
$$

where $\phi(x)$ is the angle $(0 \leqq \phi(x) \leqq \pi)$ between the radius to the identity of the group and that to the element $x$ (if $x_{1}$ is the real coordinate of $x$ then $\phi(x)$ $=$ Arccos $\left.x_{1}\right)$. The values at $\phi=0$ and $\phi=\pi$ are determined by continuity.

It is easy to show that the conjugate class of an element $x$ with real coordinate $x_{1}$ is the two-dimensional sphere consisting of all elements whose real coordinate is $x_{1}$ so that $\phi(x)$ is a central function on $Q$. Given a central function $f$ let us denote by $F$ the function on the reals modulo $\pi$ defined by

$$
F(\phi)=f(x)
$$

where $\phi=\phi(x)$. Since $\phi$ and $f$ are central functions this definition will be independent of the choice of $x$. Since translation by an element of $Q$ is simply a rotation of the three sphere it follows that the invariant measure of $Q$ is the same as the Lebesgue measure on the unit sphere in four space. We have then

$$
\int_{Q} f(x) d x=(2 / \pi) \int_{0}^{\pi} F(\phi) \sin ^{2} \phi d \phi .
$$

$(2 / \pi)$ is a normalizing factor to make the total measure of $Q$ equal one.

5. The Dirichlet kernels on $Q$. Let us order the characters on $Q$ according to degree. By Definition 2.6 and Theorem 4.1 we have for the Dirichlet kernels

$$
D_{n}(x)=(1 / \sin \phi(x)) \sum_{k=1}^{n} k \sin k \phi(x) .
$$

We shall frequently use the symbol $D_{n}$ to mean the function of $\phi$ induced by $D_{n}$ as in equation (4.3).

Let

$$
A_{n}(\phi)=1+2 \sum_{k=1}^{n} \cos k \phi=\frac{\sin (n+1 / 2) \phi}{\sin (\phi / 2)} ; A_{0}(\phi)=1 \text {. }
$$

$$
D_{n}(\phi)=(1 / 2 \sin \phi)\left[\frac{1}{2} A_{n}(\phi) \cot \frac{1}{2} \phi-\frac{(2 n+1) \cos (n+1 / 2) \phi}{2 \sin (\phi / 2)}\right] \text {. }
$$

LEMMA.

This relationship follows immediately from equation (5.2) and the observation that

$$
D_{n}(\phi)=-(1 / 2 \sin \phi) \frac{d}{d \phi} A_{n}(\phi)
$$


(5.5) TheOREM. For any positive $\epsilon$ and for $n$ sufficiently large we have

$$
(4 / \pi)+\epsilon \geqq(1 / n) \int_{Q}\left|D_{n}(x)\right| d x \geqq\left(2^{1 / 2} / \pi\right)-\epsilon .
$$

Proof.

$$
\begin{aligned}
\int_{Q} \mid & D_{n}(x) \mid d x \\
& =(2 / \pi) \int_{0}^{\pi}\left|D_{n}(\phi)\right| \sin ^{2} \phi d \phi \\
& =(1 / \pi) \int_{0}^{\pi}\left|\frac{1}{2} A_{n}(\phi) \cot \left(\frac{1}{2} \phi\right)-\frac{(2 n+1) \cos (n+1 / 2) \phi}{2 \sin (\phi / 2)}\right| \sin \phi d \phi \\
& <(1 / \pi) \int_{0}^{\pi}\left|A_{n}(\phi)\right| d \phi+\frac{2 n+1}{\pi} \int_{0}^{\pi} \cos \frac{1}{2} \phi d \phi \\
& =(1 / \pi) \int_{0}^{\pi}\left|A_{n}(\phi)\right| d \phi+(4 n / \pi)+(2 / \pi) .
\end{aligned}
$$

But for some positive constant $C$ we have (see $[6, \mathrm{p} .52]$ )

$$
\int_{0}^{\pi}\left|A_{n}(\phi)\right| d \phi \sim C \log n .
$$

So $(1 / n) \int_{Q}\left|D_{n}(x)\right| d x \leqq(4 / \pi)$. On the other hand

$$
\begin{aligned}
\int_{Q}\left|D_{n}(x)\right| d x & \\
& \geqq \frac{2 n+1}{\pi} \int_{0}^{\pi}\left|\cos \left(n+\frac{1}{2}\right) \phi\right| \cos \frac{1}{2} \phi d \phi-(1 / \pi) \int_{0}^{\pi}\left|A_{n}(\phi)\right| d \phi \\
> & \left(2^{1 / 2} / 2\right) \frac{2 n+1}{\pi} \int_{0}^{\pi / 2}\left|\cos \left(n+\frac{1}{2}\right) \phi\right| d \phi-(1 / \pi) \int_{0}^{\pi}\left|A_{n}(\phi)\right| d \phi .
\end{aligned}
$$

By making use of the periodicity of $\cos (n+1 / 2) \phi$ we obtain

$$
\begin{aligned}
\int_{Q}\left|D_{n}(x)\right| d x & >\left(2^{1 / 2} / 2\right) \frac{2 n+1}{\pi} n \int_{0}^{\pi /(2 n+1)} \cos \left(n+\frac{1}{2}\right) \phi d \phi \\
& -(1 / \pi) \int_{0}^{\pi}\left|A_{n}(\phi)\right| d \phi \\
= & \left(2^{1 / 2} n / \pi\right)-(1 / \pi) \int_{0}^{\pi}\left|A_{n}(\phi)\right| d \phi .
\end{aligned}
$$


Again making use of Equation (5.6) we have the desired result.

(5.7) Theorem. For any $x$ in $Q$ there is a continuous function whose character series diverges for that $x$.

This theorem follows immediately from Theorems 2.7 and 5.5.

6. The Fejer kernels. The Fejer kernels of the $k$ th order are the functions, $D_{n}^{\boldsymbol{k}}$, obtained from the sequence of Dirichlet kernels using Cesaro summation of the $k$ th order, that is, if we let $D_{n}^{0}=D_{n}$, then for any positive integer $k$

$$
\left(\begin{array}{c}
n+k-1 \\
k
\end{array}\right) D_{n}^{k}(x)=\sum_{\alpha=1}^{n} D_{\alpha}^{k-1}(x)
$$

or, equivalently,

$$
\left(\begin{array}{c}
n+k-1 \\
k
\end{array}\right) D_{n}^{k}(x)=\sum_{\alpha=1}^{n} r_{\alpha}\left(\begin{array}{c}
n+k-\alpha \\
k
\end{array}\right) \chi^{\alpha}(x) .
$$

(6.3) TheOREM. $\int_{G} D_{n}^{k}(x) d x=1$ on any compact group $G$ satisfying the second axiom of countability.

Proof. Since $\int_{G} \chi^{\alpha}(x) d x=0$ if $\chi^{\alpha} \not \equiv 1$ and $\int_{G} d x=1$, it follows directly from Equation (6.2) that

$$
\left(\begin{array}{c}
n+k-1 \\
k
\end{array}\right) \int_{G} D_{n}^{k}(x) d x=\left(\begin{array}{c}
n+k-1 \\
k
\end{array}\right)
$$

from which the desired result follows.

7. The Fejer kernels of the first order on $Q$.

(7.1) TheOREм. The Lebesgue constants with respect to the Fejer kernels of the first order are unbounded above, in fact $\int_{Q}\left|D_{n}^{1}(x)\right| d x \sim k \log n$ for some positive constant $k$.

Proof. It is well known that we may write

$$
\sum_{k=0}^{n} A_{k}(\phi)=F_{n}(\phi)=\frac{1-\cos (n+1) \phi}{2 \sin ^{2}(\phi / 2)} .
$$

From Equations (5.4), (6.1), and (7.2) we have

$$
\begin{aligned}
D_{n}^{1}(\phi) & =-\frac{1}{2 n \sin \phi} \frac{d}{d \phi} \sum_{k=0}^{n} A_{k}(\phi) \\
& =-\frac{1}{2 n \sin \phi} \frac{d}{d \phi} \frac{1-\cos (n+1) \phi}{2 \sin ^{2}(\phi / 2)} \\
& =-(1 / \sin \phi)\left[\frac{n+1}{n} \frac{\sin (n+1) \phi}{4 \sin ^{2}(\phi / 2)}-\frac{\cos (\phi / 2)}{2 \sin (\phi / 2)} \frac{F_{n}(\phi)}{n}\right] .
\end{aligned}
$$


Consequently

$$
\begin{aligned}
\left|D_{n}^{1}(\phi)\right| \sin ^{2} \phi & =|\sin \phi|\left|\frac{n+1}{n} \frac{\sin (n+1) \phi}{4 \sin ^{2}(\phi / 2)}-\frac{\cos (\phi / 2)}{2 \sin (\phi / 2)} \frac{F_{n}(\phi)}{n}\right| \\
& =\left|\frac{n+1}{2 n} \sin (n+1) \phi \cot (\phi / 2)-(1 / n) \cos ^{2}(\phi / 2) F_{n}(\phi)\right| \\
& =\left|\frac{n+1}{2 n}\left[A_{n+1}(\phi)-\cos (n+1) \phi\right]-(1 / n) \cos ^{2}(\phi / 2) F_{n}(\phi)\right|
\end{aligned}
$$

We have finally

$$
\begin{aligned}
\int_{Q}\left|D_{n}^{1}(x)\right| d x & =(2 / \pi) \int_{0}^{\pi}\left|D_{n}^{1}(\phi)\right| \sin ^{2} \phi d \phi \\
& =(2 / \pi) \int_{0}^{\pi} \mid \frac{n+1}{2 n}\left[A_{n+1}(\phi)-\cos (n+1) \phi\right] \\
& \quad-(1 / n) \cos ^{2}(\phi / 2) F_{n}(\phi) \mid d \phi \\
& \sim(2 / \pi) \int_{0}^{\pi}\left|A_{n+1}(\phi)\right| d \phi \sim k \log n .
\end{aligned}
$$

The last two steps follow from the fact that

$$
\int_{0}^{\pi}\left|F_{n}(\phi)\right| d \phi
$$

is uniformly bounded (see [6, p. 58]) and from (5.6).

(7.3) Theorem. For any $x$ in $Q$ there is a continuous function whose character series diverges for that $x$ even when $(C, 1)$ summability is used.

This theorem follows immediately from Theorems 7.1 and 2.7.

\section{The Fejer kernels of the third order.}

(8.1) ThEOREM. The Fejer kernels of the third order on $Q$ are positive functions.

This theorem follows immediately from a theorem of Fejer [3, Theorem 1] which asserts that the $(C, 3)$ sums of the series $\sum_{1}^{\infty} n \sin n \phi$ are positive for $0<\phi<\pi$.

(8.2) THEOREM. $D_{n}^{3}(\phi)$ converges to 0 uniformly outside of every neighborhood of the identity.

Proof. We have, from Equations (5.4) and (7.2), 


$$
\begin{aligned}
S_{n}^{1}(\phi) & =\sum_{1}^{n} D_{k}(\phi)=-(1 /(2 \sin \phi)) \frac{d}{d \phi} \sum_{0}^{n} A_{k}(\phi) \\
& =-(1 / 2 \sin \phi) \frac{d}{d \phi} \frac{1-\cos (n+1) \phi}{2 \sin ^{2}(\phi / 2)} \\
S_{n}^{2}(\phi) & =\sum_{1}^{n} S_{k}^{1}(\phi)=-(1 / 2 \sin \phi) \frac{d}{d \phi} \sum_{0}^{n} \frac{1-\cos (k+1) \phi}{2 \sin ^{2}(\phi / 2)} \\
& =-(1 / \sin \phi) \frac{d}{d \phi}\left(\frac{n+3 / 2-A_{n+1}(\phi) / 2}{4 \sin ^{2}(\phi / 2)}\right), \\
S_{n}^{3}(\phi) & =\sum_{1}^{n} S_{k}^{2}(\phi) \\
& =-(1 / \sin \phi) \frac{d}{d \phi}\left[\frac{n(n+1)+3(n+1)+1-\sum_{0}^{n+1} A_{k}(\phi)}{8 \sin ^{2}(\phi / 2)}\right] \\
& =-(1 / \sin \phi) \frac{d}{d \phi}\left\{\frac{1}{8 \sin ^{2}(\phi / 2)}\left[(n+2)^{2}-\frac{1-\cos (n+2) \phi}{2 \sin ^{2}(\phi / 2)}\right]\right\}
\end{aligned}
$$

We have finally

$$
\begin{aligned}
D_{n}^{3}(\phi) & =\left[1 /\left(\begin{array}{c}
n+2 \\
3
\end{array}\right)\right] S_{n}^{3}(\phi) \\
& =\frac{(n+2)(1-\cos \phi)[(n+2)+(1 / \sin \phi) \sin (n+2) \phi]-2[1-\cos (n+2) \phi]}{4\left(\begin{array}{c}
n+2 \\
3
\end{array}\right)(1-\cos \phi)^{3}} .
\end{aligned}
$$

We see that the theorem follows from this last expression if we make use of the fact that

$$
k \sin \phi \geqq|\sin k \phi|
$$

for all non-negative integers $k$ and for all $\phi, 0 \leqq \phi \leqq \pi$. Inequality (8.7) is easily proved by induction.

(8.8) THEOREM. The character series of a function $f$ in $L^{1}$ is $(C, 3)$ summable to the function at each point of continuity. If $f$ is continuous the character series is uniformly $(C, 3)$ summable to $f$.

Theorem 8.8 follows immediately from Theorems 8.1, 8.2, 6.3, and 2.9.

9. The Fejer kernels of the second order. Since the series $\sum_{n=1}^{\infty}(-1)^{n+1} n^{2}$ $=\lim _{\phi \rightarrow \pi} \sum_{n=1}^{\infty}(n \sin n \phi) /(\sin \phi)$ is not $(C, 2)$ summable to 0 , one might guess that Fejer's theorem must fail when $(C, 2)$ summability is used. It is interesting to note that the volume element, $\sin ^{2} \phi$, is just enough to smooth 
the kernel function down to 0 at $\pi$ and to make the analogue of Fejer's theorem true.

(9.1) THEOREM. $D_{n}^{2}(\phi) \sin ^{2} \phi$ converges uniformly to zero outside of every neighborhood of the identity.

Proof. From Equations 8.4 and 5.2 we have

$$
\begin{aligned}
D_{n}^{2}(\phi) \sin ^{2} \phi= & -\frac{\sin \phi}{\left(\begin{array}{c}
n+1 \\
2
\end{array}\right)} \frac{d}{d \phi} \frac{(2 n+3) \sin (\phi / 2)-\sin (2 n+3) \phi / 2}{8 \sin ^{3}(\phi / 2)} \\
= & \frac{n+3 / 2}{4\left(\begin{array}{c}
n+1 \\
2
\end{array}\right)} \cos (\phi / 2) \frac{\cos (\phi / 2)-\cos (2 n+3) \phi / 2}{\sin ^{2}(\phi / 2)} \\
& +\frac{3 \cos ^{2}(\phi / 2)[(2 n+3) \sin (\phi / 2)-\sin (n+3 / 2) \phi]}{8\left(\begin{array}{c}
n+1 \\
2
\end{array}\right) \sin ^{3}(\phi / 2)} .
\end{aligned}
$$

The desired result follows from Equation 9.2.

(9.3) TheOREM. $\int_{0}^{\pi}\left|D_{n}^{2}(\phi)\right| \sin ^{2} \phi d \phi$ is bounded.

Proof. Examination of Equation 9.2 shows that it will be enough to prove

$$
\begin{aligned}
& \int_{0}^{\pi / 2} \frac{|\cos x-\cos n x|}{\sin ^{2} x} d x=O(n), \\
& \int_{0}^{\pi / 2} \frac{|n \sin x-\sin n x|}{\sin ^{3} x} d x=O\left(n^{2}\right) .
\end{aligned}
$$

To prove (9.4) we note that

$$
\frac{2|\cos x-\cos n x|}{\sin ^{2} x}=\frac{|[\sin (n-1) x / 2][\sin (n+1) x / 2]|}{\sin ^{2} x} \leqq \frac{(n-1)(n+1)}{4} \text {. }
$$

We have, therefore,

$$
\begin{aligned}
\int_{0}^{\pi / 2} \frac{|\cos x-\cos n x|}{\sin ^{2} x} d x & =\int_{0}^{\pi / n}+\int_{\pi / n}^{\pi / 2} \frac{|\cos x-\cos n x|}{\sin ^{2} x} d x \\
& \leqq \frac{n^{2}-1}{8} \frac{\pi}{n}+2 \int_{\pi / n}^{\pi / 2} \frac{d x}{\sin ^{2} x} \\
& =\frac{n^{2}-1}{n} \frac{\pi}{8}-\left.2 \operatorname{ctn} x\right|_{\pi / n} ^{\pi / 2} \leqq n+2 \operatorname{ctn} \pi / n \leqq k n .
\end{aligned}
$$


To prove Equation (9.5) we consider $x, 0 \leqq x \leqq \pi / n$. We have

$$
\frac{|n \sin x-\sin n x|}{\sin ^{3} x} \leqq \frac{(1 / 3 !) n^{3} x^{3}}{\left(x-(1 / 3 !) x^{3}\right)^{3}} \leqq n^{3} .
$$

Therefore

$$
\begin{aligned}
& \int_{0}^{\pi / 2} \frac{|n \sin x-\sin n x|}{\sin ^{3} x} d x \leqq \int_{0}^{\pi / n} n^{3} d x+\int_{\pi / n}^{\pi / 2} \frac{n \sin x+1}{\sin ^{3} x} d x \\
& =\pi n^{2}-\left.n \operatorname{ctn} x\right|_{\pi / n} ^{\pi / 2}-\left.\frac{\cos x}{2 \sin ^{2} x}\right|_{\pi / n} ^{\pi / 2}+\left.\frac{1}{2} \log |\tan x / 2|\right|_{\pi / n} ^{\pi / 2} \leqq L n^{2} .
\end{aligned}
$$

(9.6) Theorem. If $f$ is continuous at a point, $x$, and is in $L^{1}$, then the character series of $f$ will be $(C, 2)$ summable to $f(x)$ at $x$. If $f$ is continuous, then the character series will be uniformly $(C, 2)$ summable.

This theorem follows immediately from Theorems 9.1, 9.3, 6.3, and 2.9.

10. The Fejer-Lebesgue theorem.

(10.1) Theorem. If $f$ is in $L^{1}$, then the character series of $f$ is $(C, 2)$ summable to $f$ almost everywhere.

Proof. The proof of this theorem is essentially classical and has been patterned after that given in [12, pp. 415-416].

By Theorem 3.2 it will be enough to prove that $\left|\int C_{s}(f, x) D_{n}^{2}(x) d x-f(s)\right|$, or equivalently, $\left|\int\left[C_{s}(f, x)-f(s)\right] D_{n}^{2}(x) d x\right|$, converges to 0 for almost all $s$. We shall need the following lemmas.

(10.2) LEMMA. $D_{n}^{2}(\phi) \leqq D_{n}^{2}(0) \leqq 2 n^{2}$.

Proof. On any compact group $D_{n}$ takes its maximum value at the identity since the characters all take their maximum value, the degree of their representation, there. The first inequality follows from this remark. The second inequality is obtained by estimating the $(C, 2)$ sums of the sequence $D_{n}(0)$ $=\sum_{1}^{n}(-1)^{k+1} k^{2}$ as follows:

$$
\begin{gathered}
S_{n}=D_{n}(0) \leqq n^{2} ; \\
S_{n}^{1} \leqq \sum_{1}^{n} k^{2} \leqq n^{3} ; \quad S_{n}^{2} \leqq \sum_{1}^{n} k^{3} \leqq n^{4} ; \quad D_{n}^{2}(0)=\frac{S_{n}^{2}}{\left(\begin{array}{c}
n+1 \\
2
\end{array}\right)} \leqq 2 n^{2} .
\end{gathered}
$$

Let $U_{d}(e)$ denote all elements, $x$, for which $\phi(x)<d$, for $d$ a positive number.

(10.3) LemMa. $\lim _{d \rightarrow 0}\left(1 / d^{3}\right) \int_{U_{d^{(e)}}}\left[C_{s}(f, x)-f(s)\right] d x=0$ for almost all $s$.

Proof. By a well known property of space differentiation (see for example [5, Theorem 18.4.1]) we have 
(10.4)

$$
\lim _{d \rightarrow 0} \frac{\int_{U_{d}(e)}\left[C_{8}(f, x)-f(s)\right] d x}{\int_{U_{d}(e)} d x}=C_{s}(f, e)-f(s)=0
$$

for almost all s. But

$$
\begin{aligned}
\int_{U_{d}(e)} d x & =(2 / \pi) \int_{0}^{d} \sin ^{2} \theta d \theta=(1 / \pi)\left[d-\frac{1}{2} \sin 2 d\right] \\
& =(1 / 2 \pi)\left[(1 / 3 !) d^{3}-(32 / 5 !) d^{5}+\cdots\right] .
\end{aligned}
$$

The lemma now follows from (10.4) and (10.5).

(10.6) Lemma. $\int_{U_{1 / n}(e)}\left[C_{s}(f, x)-f(s)\right] D_{n}^{2}(x) d x$ converges to 0 for almost all $s$.

Proof.

$$
\begin{aligned}
\left|\int_{U_{1 / n}(e)}\left[C_{s}(f, x)-f(s)\right] D_{n}^{2}(x) d x\right| & \leqq \max D_{n}^{2}(\phi)\left|\int_{U_{1 / n}(e)}\left[C_{s}(f, x)-f(s)\right] d x\right| \\
& \leqq 2 n^{2}\left|\int_{\left.U_{1 / n} e\right)}\left[C_{s}(f, x)-f(s)\right] d x\right| .
\end{aligned}
$$

This integral approaches 0 by Lemma 10.3 .

(10.7) Lemma. $\lim _{n \rightarrow \infty} \int_{C_{\left(U_{d}(e)\right)}}\left[C_{s}(f, x)-f(s)\right] D_{n}^{2}(x) d x$ is equal to 0 for almost all s.

This lemma follows immediately from Theorem 9.1.

(10.8) Lemma. For $0<d<(\pi / 2), \int_{U_{d}(e)-U_{1 / n}(e)}\left[C_{8}(f, x)-f(s)\right] D_{n}^{2}(x) d x$ converges to 0 for almost all $s$.

Proof. For $0 \leqq \phi \leqq d<\pi / 2$ we may show

$$
D_{n}^{2}(\phi) \leqq K \frac{2 n+3}{\left(\begin{array}{c}
n+1 \\
2
\end{array}\right) \sin ^{4}(\phi / 2)}
$$

for some positive constant $K$ as follows:

$$
\begin{aligned}
D_{n}^{2}(\phi)= & \frac{3 \cos ^{2}(\phi / 2)[(2 n+3) \sin (\phi / 2)-\sin (2 n+3)(\phi / 2)]}{8\left(\begin{array}{c}
n+1 \\
2
\end{array}\right) \sin ^{3}(\phi / 2) \sin ^{2} \phi} \\
& -\frac{(n+3 / 2)}{4\left(\begin{array}{c}
n+1 \\
2
\end{array}\right)} \cos (\phi / 2) \frac{\cos (\phi / 2)-\cos (2 n+3)(\phi / 2)}{\sin ^{2}(\phi / 2) \sin ^{2} \phi}
\end{aligned}
$$




$$
\begin{aligned}
& \leqq \frac{3 \sin (\phi / 2)[(2 n+3)-\csc (\phi / 2) \sin (2 n+3)(\phi / 2)]}{8\left(\begin{array}{c}
n+1 \\
2
\end{array}\right) \sin ^{3}(\phi / 2) \sin ^{2} \phi} \\
& +\frac{2(n+3 / 2)}{4\left(\begin{array}{c}
n+1 \\
2
\end{array}\right) \sin ^{2}(\phi / 2) \sin ^{2} \phi} \\
& \leqq \frac{8(2 n+3)}{8\left(\begin{array}{c}
n+1 \\
2
\end{array}\right) \sin ^{2}(\phi / 2) \sin ^{2} \phi}+\frac{4\left(\begin{array}{c}
n+1 \\
2
\end{array}\right) \sin ^{2}(\phi / 2) \sin ^{2} \phi}{2 n+3}
\end{aligned}
$$

Since

$$
\begin{aligned}
\int_{1 / n}^{d}\left|C_{8}(f, \phi)-f(s)\right| D_{n}^{2}(\phi) & \sin ^{2}(\phi) d \phi \\
& \leqq \frac{K(2 n+3)}{\left(\begin{array}{c}
n+1 \\
2
\end{array}\right)} \int_{0}^{d}\left|C_{s}(f, \phi)-f(s)\right| \frac{\sin ^{2} \phi}{\sin ^{4}(\phi / 2)} d \phi
\end{aligned}
$$

and

$$
\frac{K(2 n+3)}{\left(\begin{array}{c}
n+1 \\
2
\end{array}\right)} \int_{0}^{d}\left|C_{s}(f, \phi)-f(s)\right|\left|\frac{1}{\sin ^{4}(\phi / 2)}-\frac{16}{\phi^{4}}\right| \sin ^{2} \phi d \phi
$$

approaches zero as $n$ approaches infinity it will be enough to prove that

$$
\frac{2 n+3}{\left(\begin{array}{c}
n+1 \\
2
\end{array}\right)} \int_{1 / n}^{d} \frac{\left|C_{s}(f, \phi)-f(s)\right|}{\phi^{4}} \sin ^{2} \phi d \phi \text { approaches zero. }
$$

Let $F(\alpha)=\int_{0}^{\alpha}\left|C_{s}(f, \phi)-f(s)\right| \sin ^{2} \phi d \phi$. By integration by parts we have

$$
\begin{aligned}
\int_{1 / n}^{d} \frac{\left|C_{s}(f, \phi)-f(s)\right|}{\phi^{4}} & \sin ^{2} \phi d \phi \\
& =\left(1 / d^{4}\right) F(d)-n^{4} F(1 / n)+4 \int_{1 / n}^{d} \frac{F(\phi)}{\phi^{5}} d \phi .
\end{aligned}
$$


But by Lemma 10.3 for any positive number, $b$, we may choose a positive number $\delta=\delta(b)<d$ for which $\left(1 / \phi^{3}\right) F(\phi)<b$ if $0<\phi \leqq \delta$. Equation 10.9 then gives us

$$
\int_{1 / n}^{\delta} \frac{\left|C_{s}(f, \phi)-f(s)\right|}{\phi^{4}} \sin ^{2} \phi d \phi<(b / \delta)+4 b \int_{1 / n}^{\delta} \frac{d \phi}{\phi^{2}} \leqq(b / \delta)+4 b n,
$$

and consequently

$$
\begin{aligned}
J_{n} & \equiv \frac{2 n+3}{\left(\begin{array}{c}
n+1 \\
2
\end{array}\right)} \int_{1 / n}^{\delta} \frac{\left|C_{s}(f, \phi)-f(s)\right|}{\phi^{4}} \sin ^{2} \phi d \phi \\
& <(b / \delta) \frac{2 n+3}{\left(\begin{array}{c}
n+1 \\
2
\end{array}\right)}+4 b n \frac{2 n+3}{\left(\begin{array}{c}
n+1 \\
2
\end{array}\right)} .
\end{aligned}
$$

So $\lim \sup _{n \rightarrow \infty} J_{n} \leqq 16 b$. Since $b$ may be chosen arbitrarily small the right-hand side of the above inequality approaches zero as $n$ approaches infinity. The desired result now follows from the fact that $\int_{\delta}^{d}\left[C_{s}(f, \phi)-f(s)\right] D_{n}^{2}(\phi) \sin ^{2} \phi d \phi$ approaches 0 by Theorem 9.1 .

Theorem 10.1 is an immediate consequence of Lemmas 10.6, 10.7, and 10.8 .

11. Kolmogoroff's theorem. If $f$ is a function in $L^{2}$ on the circle and $\left(n_{k}\right)$ is a sequence of integers with the property that $n_{k+1} / n_{k}>C>1$, then the subsequence $S_{n_{k}}$ of the sequence of partial sums of the Fourier series of $f$ will converge almost everywhere. This theorem was first proved by Kolmogoroff. A proof by Marcinkiewicz [6, Theorem 83] shows that this is a simple consequence of the Fejer-Lebesgue theorem. In the group $Q$ we may prove essentially the same theorem, although, because we have $(C, 2)$ convergence in the Fejer-Lebesgue theorem instead of $(C, 1)$ convergence, we shall need a lemma due to W. Meyer-König [8, Satz 2].

(11.1) Lemma. If $\left(a_{n}\right)$ is a numerical sequence with Ostrowski gaps (i.e. there exists two increasing sequences of integers $\left(n_{i}\right)$ and $\left(n_{i}^{\prime}\right)$ such that $n_{i}^{\prime} / n_{i}$ $>C>1, n_{i}^{\prime}<n_{i+1}$, and $a_{n}$ is zero if $\left.n_{i} \leqq n \leqq n_{i}^{\prime}\right)$ and if the series $\left(a_{n}\right)$ is $(C, k)$ summable to sfor some positive integer $k$, then any subsequence $\left(s_{m_{j}}\right)$ of the partial sums of the $\left(a_{n}\right)$, where $n_{i} \leqq m_{j} \leqq n_{i}^{\prime}$, converges to $s$.

(11.2) Theorem (Kolmogorofr). If $f$ is a function of $L^{2}$ on the group $Q$ and $\left(n_{k}\right)$ is a sequence of integers such that $n_{k+1} / n_{k}>C>1$ then the subsequence $\left(S_{n_{k}}\right)$ of the sequence of partial sums of the character series of $f$ will converge to $f$ almost everywhere.

Proof. If the character series of $f$ has Ostrowski gaps, then the theorem is a direct consequence of Theorem 10.1 (the Fejer-Lebesgue theorem on $Q$ ) 
and Lemma 11.1. If $f$ does not have Ostrowski gaps in its character series, then we consider the two character series

$$
\begin{aligned}
& f_{1} \sim A^{1}+A^{2}+A^{5}+A^{6}+A^{7}+A^{8}+A^{13}+\cdots+A^{20}+\cdots, \\
& f_{2} \sim A^{3}+A^{4}+A^{9}+A^{10}+A^{11}+A^{12}+A^{21}+\cdots+A^{29}+\cdots
\end{aligned}
$$

where

$$
f \sim\left(r_{i j}^{n} a_{i j}^{n} m_{i j}^{n}\right)=\left(A^{n}\right) .
$$

Since $f$ is in $L^{2}$ it follows from Parseval's equation that $f_{1}$ and $f_{2}$ are in $L^{2}$; consequently, since $f_{1}$ and $f_{2}$ have Ostrowski gaps their character series converge to them almost everywhere. Since $f$ is their sum we have the same result for $f$.

12. Abel summability at a point of continuity. Although Abel summability at a point of continuity follows from the analogue of Fejer's theorem, it is of interest to give an independent proof of this fact.

Let $A_{z}(\phi)=\sum_{n=1}^{\infty} n(\sin n \phi / \sin \phi) z^{n-1}$ for $0 \leqq \phi \leqq \pi$ and $0 \leqq z<1$.

(12.1) Theorem. For $f$ in $L^{2}$ we have $\lim _{z \rightarrow 1} f * A_{z}(x)=f(x)$ if $x$ is a point of continuity of $f$. If $f$ is continuous then $f * A_{z}$ approaches $f$ uniformly as $z$ approaches 1.

Proof. The theorem follows from the following lemmas.

(12.2) Lemma. $A_{z}(\phi)=\left(1-z^{2}\right) /\left(1-2 z \cos \phi+z^{2}\right)^{2}$.

(12.3) Lemma. $A_{z}$ converges to zero uniformly outside of every neighborhood of the identity.

(12.4) Lemma. (2/ $\pi) \int_{0}^{\pi} A_{z}(\phi) \sin ^{2} \phi d \phi=1$.

From 12.2 and 12.4 it follows that $A_{z}$ is positive for $0 \leqq z<1$ and that $\int_{Q} A_{z}(\phi(x)) d x=1$. Theorem 12.1 then follows from Lemma 12.3 and Theorem 2.9.

Lemma 12.4 is noted in [1, p. 102, formula 7]. Lemma 12.3 is an immediate consequence of Lemma 12.2. To prove 12.2 we note that

$$
\begin{aligned}
A_{z}(\phi) & =\sum_{n=1}^{\infty} \frac{n \sin n \phi}{\sin \phi} z^{n-1}=\sum_{n=1}^{\infty} \frac{n e^{i n \phi}-e^{-i n \phi}}{2 i \sin \phi} z^{n-1} \\
& =\frac{\partial}{\partial z} \sum_{n=1}^{\infty}(1 / 2 i \sin \phi)\left\{e^{i n \phi_{Z^{n}}}-e^{-i n \phi_{z^{n}}}\right\} \\
& =\frac{\partial}{\partial z} \sum_{n=1}^{\infty}(1 / 2 i \sin \phi)\left\{\frac{e^{i \phi_{z}}}{1-e^{i \phi_{z}}}-\frac{e^{-i \phi_{z}}}{1-e^{-i \phi_{z}}}\right\} \\
& =\frac{1-z^{2}}{\left(1-2 z \cos \phi+z^{2}\right)^{2}} .
\end{aligned}
$$


13. The localization theorem. One might think that the localization theorem could be invoked to dispense with the assumption that $f$ is in $L^{2}$ in the proof of Theorem 12.1. Consequently it should be noted that the localization theorem does not hold. Let us define the function $f$ on $Q$ as follows

$$
f(x)=\left\{\begin{array}{lll}
0 & \text { if } & 0 \leqq \phi(x)<\pi / 2, \\
1 & \text { if } & \pi / 2 \leqq \phi(x) \leqq \pi
\end{array}\right.
$$

(13.1) THEOREM. The character series of the function $f$ defined above diverges at the identity of the group.

Proof. This theorem is proved simply by calculating the coefficients of the character series of $f$. We have

$$
\begin{aligned}
\int_{Q} f(x)\left[\chi_{n}(x)\right]^{c} d x & =(2 / \pi) \int_{\pi / 2}^{\pi} \frac{n \sin n \phi}{\sin \phi} \sin ^{2} \phi d \phi \\
& = \begin{cases}0 \text { for } n \text { odd and greater than } 1,1 / 2 \text { for } n=1, \\
\frac{2 n^{2}}{\pi\left(n^{2}-1\right)} \text { for } n \equiv 0 \bmod 4, \\
\frac{-2 n^{2}}{\pi\left(n^{2}-1\right)} \text { for } n \equiv 2 \bmod 4,\end{cases}
\end{aligned}
$$

and, finally,

$$
f(e) \sim(2 / \pi)\left[\frac{\pi}{4}+\frac{-2^{2}}{2^{2}-1}+\frac{4^{2}}{4^{2}-1}+\frac{-6^{2}}{6^{2}-1}+\cdots\right]
$$

which is a divergent series.

\section{BibliograPhy}

1. D. Bierens de Haan, Nouvelles tables d'intégrales définies, ed. of 1867, New York, Stechert, 1939.

2. K. Chandrasekharan and S. Minakshisundaram, Some results on double Fourier series, Duke Math. J. vol. 14 (1947) pp. 731-753.

3. L. Fejer, Neue Eigenschaften der Mittelwerte bei den Fourierreihen, J. London Math. Soc. vol. 8 (1933) pp. 53-62.

4. N. J. Fine, On the Walsh functions, Trans. Amer. Math. Soc. vol. 65 (1949) pp. 372-414.

5. H. Hahn and A. Rosenthal, Set functions, Albuquerque, University of New Mexico Press, 1948.

6. G. H. Hardy and W. W. Rogosinski, Fourier series, 2d ed., Cambridge University Press, 1950.

7. H. Hopf and H. Samelson, Ein Satz ïber die Wirkungsräume geschlossener Liescher Gruppen, Comment. Math. Helv. vol. 13 (1940-41) pp. 240-251.

8. W. Meyer-König, Limitierungsumkehrsätze mit Luckenbedingungen, I, Math. Zeit. vol. 45 (1940) pp. 447-478.

9. L. Pontrjagin, Topological groups, Princeton University Press, 1939. 
10. E. Stiefel, Kristallogphische Barestimmung der Charaktere der geschlossenen Lie'schen Gruppen, Comment. Math. Helv. vol. 17 (1944-45) pp. 165-200.

11. G. Szegö, Orthogonal polynomials, Amer. Math. Soc. Colloquium Publications, vol. 23, 1939.

12. E. C. Titchmarsh, The theory of functions, London, Oxford University Press, 1950.

13. J. L. Walsh, A closed set of normal orthogonal functions, Amer. J. Math. vol. 45 (1923) pp. 5-24.

14. A. Weil, L'intégration dans les groupes topologiques et ses applications, Paris, Hermann, 1940.

UNIVERSITY OF Michigan,

AnN Arbor, Mich.

Purdue University,

LAFAYETTE, IND. 